\title{
Physiological roles of cGMP-gated channels: lessons from mouse models and human channelopathies Martin Biel ${ }^{* 1}$, Stylianos Michalakis ${ }^{1}$, Xiangang Zong ${ }^{1}$, Elvir Becirovic ${ }^{1}$ and Alexander Pfeifer ${ }^{2}$
}

\author{
Address: ${ }^{1}$ Center for Integrated Protein Science CIPS-M and Zentrum für Pharmaforschung - Department Pharmazie, Ludwig-Maximilians- \\ Universität München, Germany and 2Institut für Pharmakologie und Toxikologie, Universität Bonn, Germany \\ Email: Martin Biel* - mbiel@cup.uni-muenchen.de \\ * Corresponding author
}

from 4th International Conference of cGMP Generators, Effectors and Therapeutic Implications

Regensburg, Germany. 19-21 June 2009

Published: II August 2009

BMC Pharmacology 2009, 9(Suppl I):S5 doi:10.1186/147I-2210-9-SI-S5

This abstract is available from: http://www.biomedcentral.com//47I-22I0/9/SI/S5

(C) 2009 Biel et al; licensee BioMed Central Ltd.

\section{Background}

Cyclic nucleotide-gated (CNG) channels are members of the superfamily of pore loop cation channels that are activated by the binding of cGMP or cAMP. The channels are important cellular switches which transduce changes in intracellular levels of cyclic nucleotides into changes of the membrane potential and the $\mathrm{Ca}^{2+}$ concentration. CNG channels play a central role in the signal transduction pathways of vision and olfaction. We have studied the physiological significance of a particular member of the CNG channel family, CNGB1. The two splicing variants of CNGB1, termed CNGB1a and CNGB1b, assemble with CNGA1 and CNGA2/4 to form the native tetrameric CNG channels of rod photoreceptors and olfactory neurons, respectively.

\section{Results}

Using gene knockout in mice we have analyzed the specific physiological roles of the two splicing variants of CNGB1 in vision and olfaction. We find that CNGB1 is not only modulating the biophysical properties of the CNG channel but that it is also crucially required for normal CNG channel targeting to outer segments and olfactory cilia. In the retina, impairment of CNG channel targeting induces a degenerative process that is reminiscent of human retinitis pigmentosa. A few years ago, a mutation in the cGMP-binding domain of CNGB1 (CNGB1aG993V) was detected in a family suffering from retinitis pigmentosa [1]. Using a combination of electrophysiologcal measurements, biochemistry and studies in mouse models we have analyzed the pathology of this channelopathy.

\section{Conclusion}

Our findings provide new insights into the functional role of distinct CNGB1 channel domains.

\section{References}

I. Bareil C, Hamel CP, Delague V, Arnaud B, Demaille J, Claustres M: Segregation of a mutation in CNGB I encoding the beta-subunit of the rod cGMP-gated channel in a family with autosomal recessive retinitis pigmentosa. Hum Genet 200I, 108:328-334. 